\title{
MOVEMENT OF SMALL AMPLITUDE PARTS IN A COUPLED CHAOTIC SYSTEM.
}

\section{Yasuteru Hosokawa}

\author{
Department of Information Science, \\ Shikoku University \\ 123-1 Furukawa, Ohjin, Tokushima, Japan \\ Email: hosokawa@keiei.shikoku-u.ac.jp
}

\begin{abstract}
We investigate the movement of small amplitude parts in a coupled chaotic system. The system consists of chaotic circuits using CMOS ring oscillators and capacitors as coupling elements. In the case of coupling 25 chaotic circuits, irregular movements of small amplitude parts are observed for the same parameter and different initial values. This is a kind of spatio-temporal phenomena. We focus attention on difference of the movement. Distributions of the small amplitude parts are investigated.
\end{abstract}

\section{INTRODUCTION}

In our past study, we have investigated a chaotic circuit using CMOS ring oscillators[1]. The circuit consists of two CMOS ring oscillators and two diodes. A CMOS ring oscillator is used for the CMOS process performance test. This oscillator consists of a series of inverter circuits. It has a very simple structure. Therefore, it is considered that the proposed circuit is realized very easily. In the past study, we have shown the bifurcation scenario, have calculated the Lyapunov exponents, have explained physical mechanism of chaos generation and so on.

In this study, we investigate the movement of small amplitude parts in a coupled chaotic system using the chaotic circuits. The study of large coupled chaotic systems is very important not only as models of nonlinear systems with many degrees of freedom but also to understand biological information processing and for engineering applications. Especially, we consider that it is important to use continuous-time chaotic circuits, which is natural real physical systems, in these studies. On the other hand, the system consists of many chaotic circuits using CMOS ring oscillators. Because this system utilizes the circuits designed by the integrated circuit technology, this study is very important for the analysis and applications of large coupled chaotic systems[2]-[5]. We consider that large coupled systems should be realized as VLSI for future studies and future applications. The proposed system is very suitable for integrated circuits. It means that VLSI realization of the system is easily. In Sec. 2, the chaotic circuit and its simplified model is described. In Sec. 3, the coupled system and the simulated results are shown. Some concluding remarks are presented in Sec. 4.

\section{CHAOTIC CIRCUIT USING CMOS RING OSCILLATORS}

Several chaotic circuits have been realized as integrated circuits. These circuits are not complex so far as an electric circuit are concerned. However, the realized circuits on the IC chip have complex

\section{Yoshifumi Nishio}

\author{
Department of Electrical and Electronics \\ Engineering, Tokushima University \\ 2-1 Minami-Josanjima, Tokushima, Japan \\ Email: nishio@ee.tokushima-u.ac.jp
}

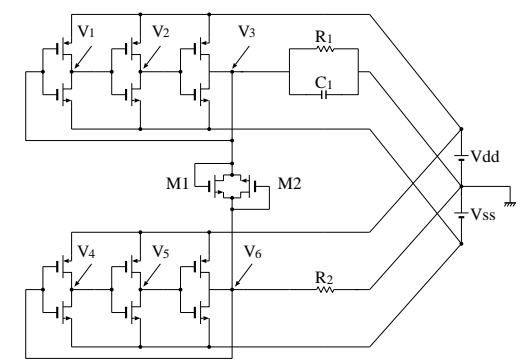

Figure 1: Chaotic circuit using ring oscillators.

structures. The basic problem is that the circuits are analog circuits and include nonlinear elements. Therefore, integration techniques are needed. One of the attraction of chaos is that a simple system generates complex phenomena. We went back again to this attraction and a simple integrated circuit was proposed. We consider utilizing a ring oscillator which has a simple structure on the chip. A CMOS ring oscillator is one of the simplest circuit constructed on the chip. The oscillator consists of the odd number of CMOS inverters. We designed a chaotic circuit using the ring oscillators. Figure 1 shows the designed circuit. In order to control amplitudes of oscillators, $R_{1}$ and $R_{2}$ are connected. The frequency of the upper side oscillator are controlled by $C_{1}$. Two three-dimensional autonomous oscillatory circuits are coupled by two diodes. We approximate $M 1, M 2$ and CMOS inverters in the circuit as follows.

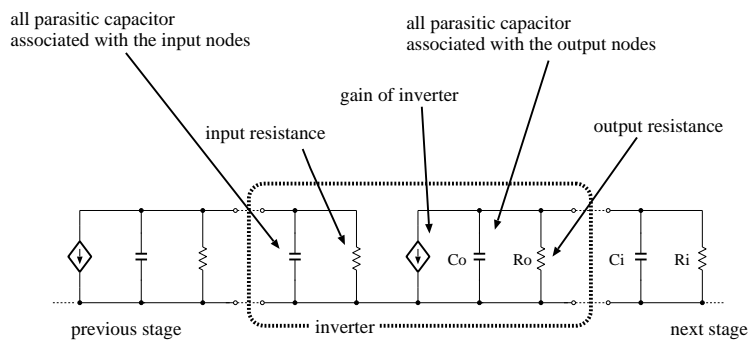

Figure 2: Model of a CMOS inverter in the ring oscillator.

We focus attention on one inverter as shown in Fig. 2. All parasitic capacitors associated with the input node is connected parallel with all parasitic capacitors associated with the output node of the previ- 


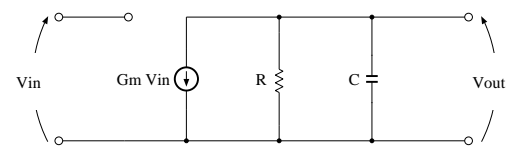

Figure 3: Simplified model of a CMOS inverter in the ring oscillator.

ous stage inverter. The input resistor is connected parallel with the output resistor of the previous stage inverter. Correspondingly, All parasitic capacitors associated with the output node is connected parallel with all parasitic capacitors associated with the input node of the next stage inverter. The output resistor is connected parallel with the input resistor of the next stage inverter. Therefore, we can obtain a simplified inverter model as shown in Fig. 3. $M 1$ and $M 2$

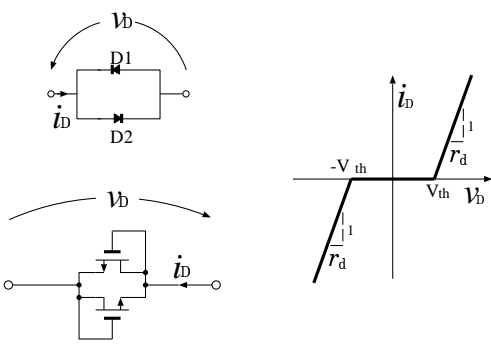

Figure 4: Diodes model.

have a characteristic of the same as the coupling diodes shown in Fig. 4. Therefore, $M 1$ and $M 2$ are approximated as the following three-regions piecewise-linear function.

$$
i_{D}=\left\{\begin{array}{cl}
\frac{1}{r_{d}}\left(v_{D}-V_{t h}\right) & \text { for } v_{D}>V_{t h} \\
0 & \text { for }-V_{t h} \leq v_{D} \leq V_{t h}, \\
\frac{1}{r_{d}}\left(v_{D}+V_{t h}\right) & \text { for } v_{D}<-V_{t h} .
\end{array}\right.
$$

Normalized circuit equations are described as follows:

$$
\left\{\begin{array}{l}
\dot{x}_{1}=-x_{1}-\alpha x_{3}, \\
\dot{x}_{2}=-x_{2}-\alpha x_{1}, \\
\dot{x}_{3}=-\beta(\gamma+1) x_{3}-\alpha \beta x_{2}-\beta \delta y_{d}, \\
\dot{x}_{4}=-x_{4}-\alpha x_{6}, \\
\dot{x}_{5}=-x_{5}-\alpha x_{4}, \\
\dot{x}_{6}=-(\varepsilon+1) x_{6}-\alpha x_{5}+\delta y_{d},
\end{array}\right.
$$

where

$$
\begin{gathered}
y_{d}=\left\{\begin{array}{cl}
x_{3}-x_{6}-1 & \text { for } x_{3}-x_{6}>1, \\
0 & \text { for }-1 \leq x_{3}-x_{6} \leq 1, \\
x_{3}-x_{6}+1 & \text { for } x_{3}-x_{6}<-1 .
\end{array}\right. \\
x_{n}=\frac{v_{n}}{V_{t h}}, \quad y_{d}=\frac{R_{d}}{V_{t h}} i_{d}, \quad \tau=\frac{1}{R C} t, \\
\alpha=G m R, \quad \beta=\frac{C}{C+C_{1}}, \quad \gamma=\frac{R}{R_{1}},
\end{gathered}
$$

\section{COUPLED SYSTEM OF THE CHAOTIC CIRCUITS}

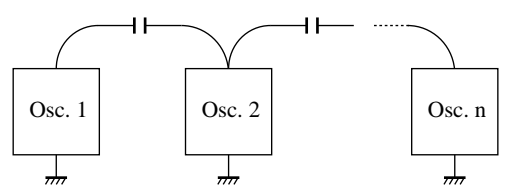

Figure 5: Coupled system of the chaotic circuits.

The circuits are coupled by capacitors as shown in Fig. 5 . The coupling points are $V_{3}$ of Fig. 1. The value of all coupling capacitors is $C_{c}$. Coupled system equations are the same as Eq. (2) except the third equation. Figure 6 shows attractors of the single

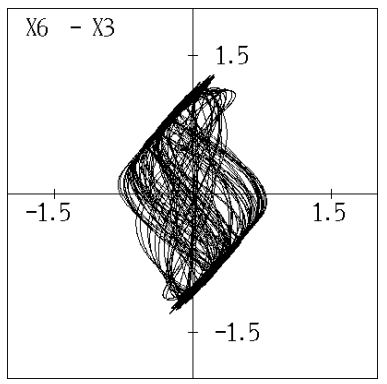

(a)

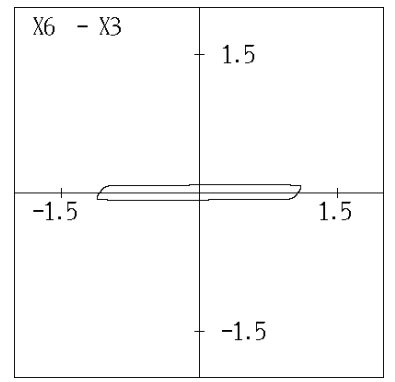

(b)
Figure 6: Attractor of the chaotic circuit using the ring oscillators. $\alpha=4, \beta=0.1, \gamma=4.5, \delta=70$ and $\varepsilon=2.4$

chaotic circuit using the ring oscillators. Chaotic attractor is observed in Fig. 6 (a). Periodic orbit is observed in Fig. 6 (b). The same parameters are used, but different initial values are used. The coupled system is simulated using the same parameters as Fig. 6. The number of the coupled chaotic circuits is 25 . At first, we investigate time series of $x_{3}$ for various sets of initial values. Figures 7 11 show simulated results of the coupled system. The horizontal axis shows time. The vertical axis shows $x_{k 3}(k=1,2, \cdots 25)$ of each chaotic circuit.

We found that the system produce one or more small amplitude parts consisting of the attractor in Fig. 6 (b). One small amplitude part is observed in Fig. 7. The position of this part is changing irregularly. In Fig. 8, all chaotic circuits generate the small amplitudes. Three small amplitude parts are observed in Fig. 9. These parts do not move. In the case that the number of coupled chaotic circuits is 25 , four or more small amplitude parts are not observed. This result means that about seven chaotic circuits are needed in order to produce one small amplitude part. Two small amplitude parts are observed in Fig. 10. Basically, this state is almost stable. However, the position of these parts rarely moves irregularly as shown in Fig. 11. In the case that initial values of all chaotic circuits are the same, one small amplitude part is observed as Fig. 7 via no small amplitude part state. Therefore, it is considered that the system wishes to produce the small amplitude parts. From these results, we can consider that each small amplitude part hates getting close.

Next, the number of the local maximum of the small amplitude are counted. This counting means calculating the distributions of 
the small amplitude parts of each oscillator. Figures 12-15 show the numbers of the local maximum of the small amplitude parts corresponding to Figs. 7-11. The horizontal axis shows the number of the chaotic circuits. The vertical axis shows the numbers of the local maximum of the small amplitude parts. Figure 12 shows that the small amplitude part is almost between no. 11 and no. 15 . Figure 13 shows that the small amplitude part is pervasive. Figure 14 shows that three small amplitude parts are observed. The center part is symmetric. However, both side parts are asymmetric. Figure 15 shows that two small amplitude parts are observed. The main parts of the small amplitude are no. 6 and no. 20. Between no. 6 and no. 20, only few counts are observed. We think that this counts corresponds to the irregular movement as shown in Fig. 11. This result shows that the irregular movement as is very rare.

\section{CONCLUSIONS}

In this study, we have investigated a coupled chaotic system utilizing CMOS ring oscillators. The system can be constructed by simple CMOS elements. We carried out computer simulation for many sets of initial values. As a result, the characteristics of this system are described as follows. The system wishes to produce one or more small amplitude parts. The existence of one small amplitude part needs about seven oscillators. Each small part hates getting close. We observed movements of the small amplitude part. We consider that the chaotic behavior of each circuit makes changing the position of the small amplitude parts. However, by the reason that one small amplitude part needs about seven chaotic circuits and each part hates getting close, in the case of many small amplitude parts we could not observed changing the position of the parts. These results contribute to the studies on VLSI realization and applications of large coupled chaotic systems.

\section{REFERENCES}

[1] Y. Hosokawa, Y. Nishio and A. Ushida, "Analysis of Chaotic Circuit Using Two Simple Ring Oscillators Coupled by Diodes," Proc. of NOLTA2001, vol. 1, pp. 203-206, Oct. 2001.

[2] K. Kaneko, “ Theory and Applications of Coupled Map Lattices," John Wiley \& Sons, 1993.

[3] A. L. Zheleznyak and L. O. Chua, "Coexistence of Low- and High-Dimensional Spatiotemporal Chaos in a Chain of Dissipatively Coupled Chua's Circuits," Int. J. Bifurcation and Chaos, vol. 4, no. 3, pp. 639-674, 1994.

[4] Y. Nishio and A. Ushida, "Spatio-Temporal Chaos in Simple Coupled Chaotic Circuits," IEEE Trans. on Circuits and Systems I, vol. 42, no. 10, pp. 678-689, Oct. 1995.

[5] M. Miyamura, Y. Nishio and A. Ushida, "Clustering in Globally Coupled System of Chaotic Circuits," Proc. of ISCAS'02, vol. 3, pp. 57-60, May 2002.

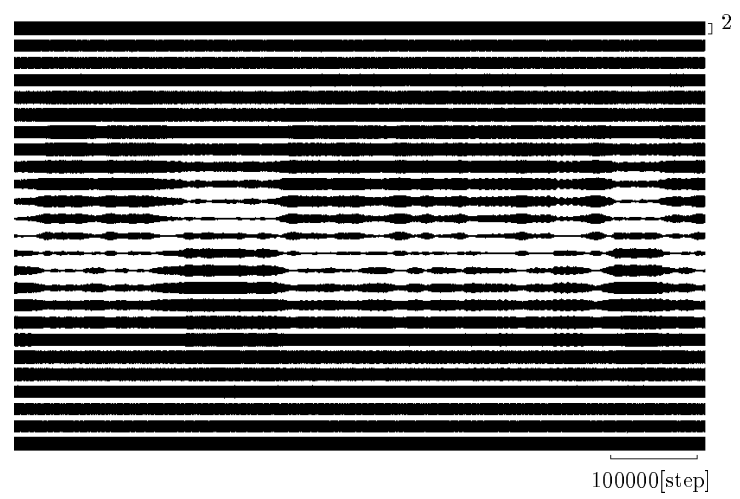

Figure 7: Simulated result in the case that one small amplitude part is observed.

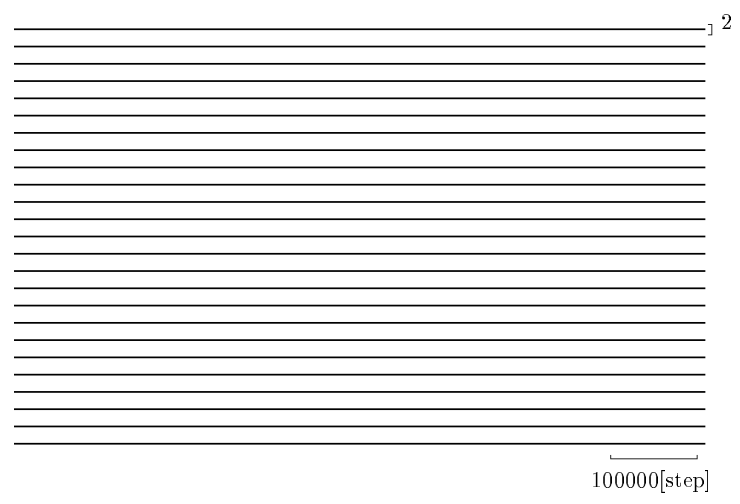

Figure 8: Simulated result in the case that all oscillators generate small amplitudes as Fig. 6 (b).

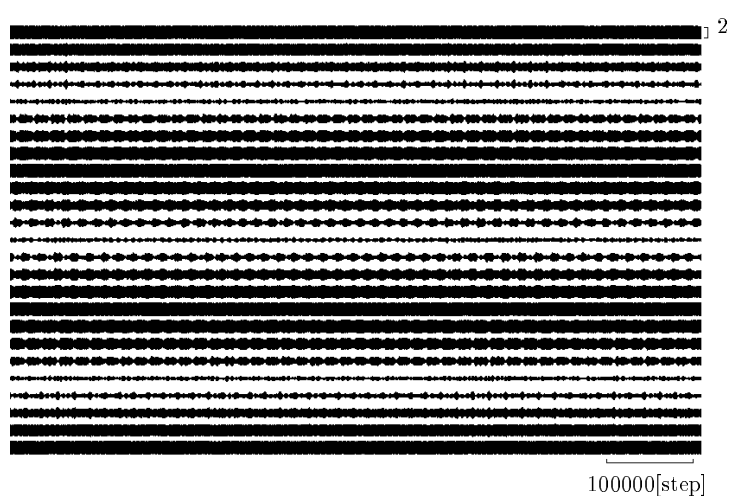

Figure 9: Simulated result in the case that three small amplitude parts are observed. 


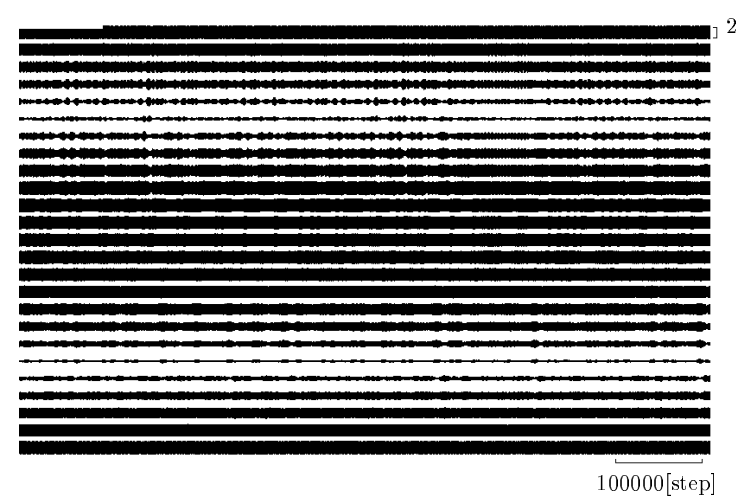

Figure 10: Simulated result in the case that two small amplitude parts moves irregularly.

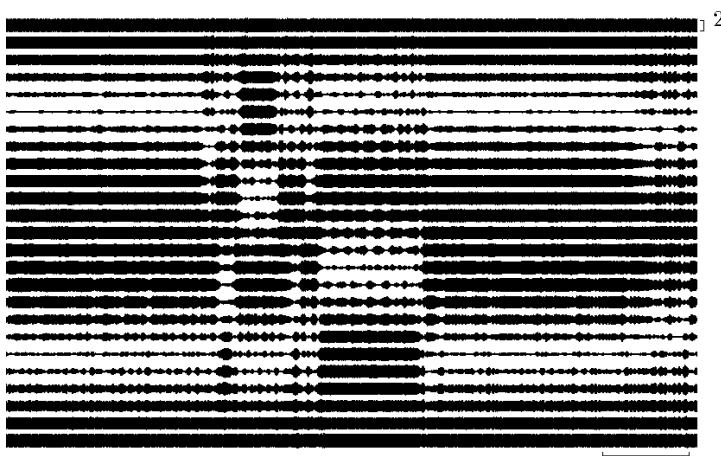

$100000[$ step]

Figure 11: Simulated result in the case of two small amplitude parts.(continued from Fig. 10)

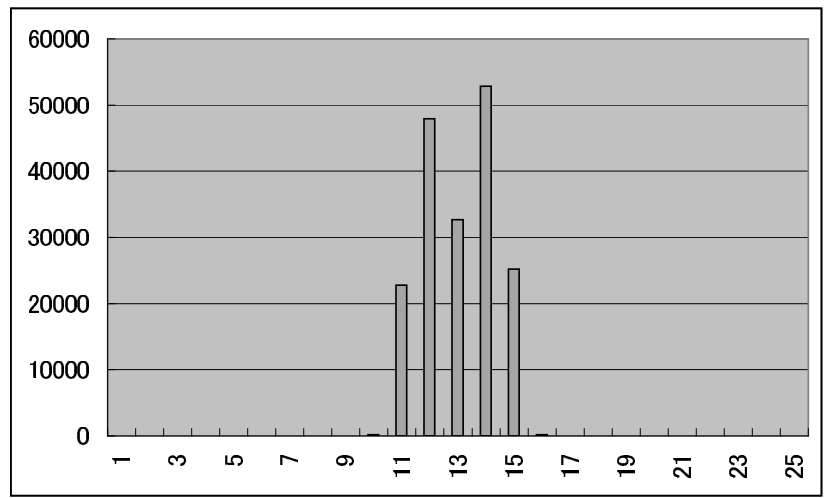

Figure 12: Number of local maximum of small amplitude in the case that one small amplitude part is observed.

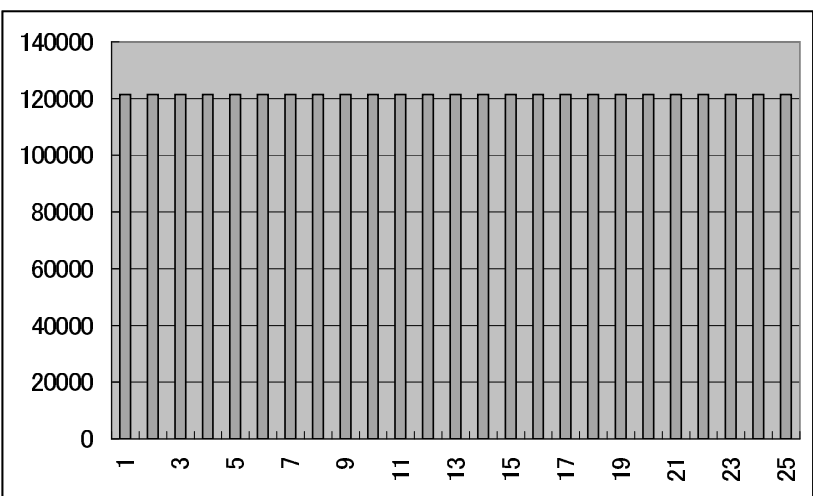

Figure 13: Number of local maximum of small amplitude in the case that all oscillators generate small amplitudes as Fig. 6 (b).

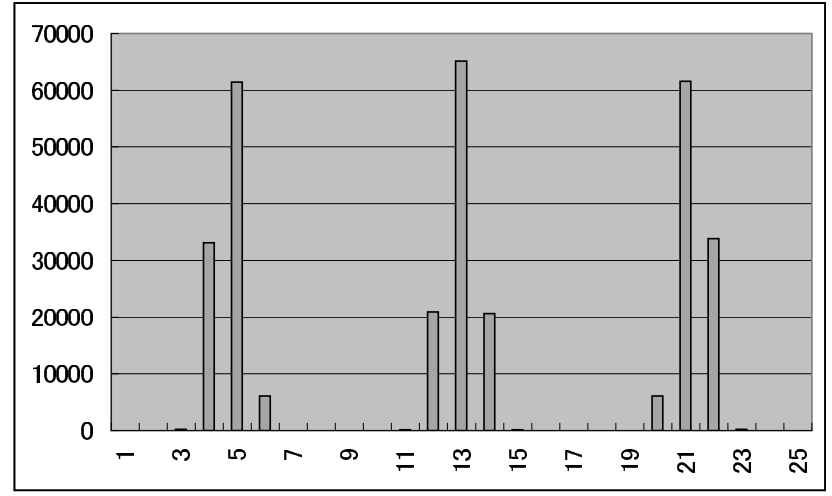

Figure 14: Number of local maximum of small amplitude in the case that three small amplitude parts are observed.

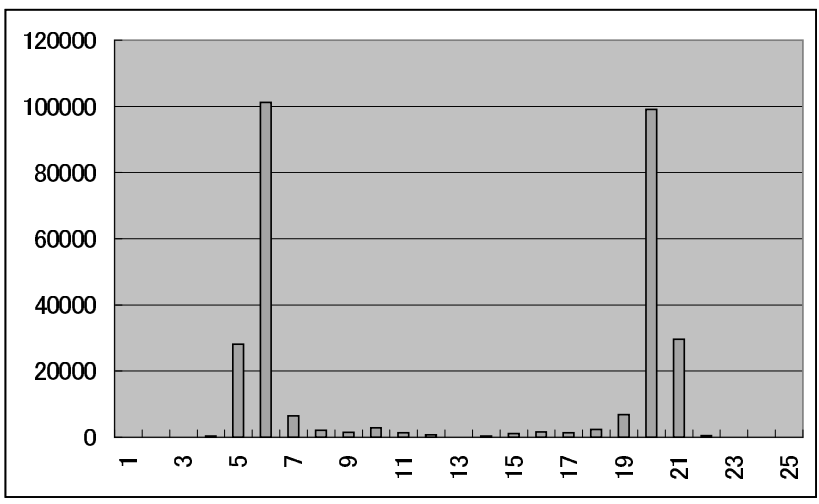

Figure 15: Number of local maximum of small amplitude in the case that two small amplitude parts moves irregularly. 